\title{
$24 \mathrm{GHz}$ LTCC Fractal Antenna Array SoP with Integrated Fresnel Lens
}

\author{
F. A. Ghaffar, M. U. Khalid, K. N. Salama, SeniorMember, IEEE, A. Shamim, Member, IEEE
}

\begin{abstract}
A novel 24 GHz mixed LTCC tape based System-onPackage (SoP) is presented which incorporates a fractal antenna array with an integrated grooved Fresnel lens. The four element fractal array employs a relatively low dielectric constant substrate $\left(\mathrm{CT707}, \varepsilon_{\mathrm{r}}=6.4\right)$ where as the lens has been realized on a high dielectric constant superstrate $\left(\mathrm{CT765}, \varepsilon_{\mathrm{r}}=68.7\right)$. The two (substrate and superstrate) are integrated through four corner posts to realize the required air gap (focal distance). The fractal array alone provides a measured gain of $8.9 \mathrm{dBi}$. Simulations predict that integration of this array with the lens increases the gain by $6 \mathrm{~dB}$. Measurements reveal that the design is susceptible to LTCC fabrication tolerances. In addition to high gain, the SoP provides a bandwidth of $8 \%$. The high performance and compact size $(24 \mathrm{~mm} \times 24 \mathrm{~mm} \times 4.8 \mathrm{~mm})$ of the design makes it highly suitable for emerging wireless applications such as automotive radar front end.
\end{abstract}

Index Terms-Fractal, Fresnel Lens, LTCC, SoP

\section{INTRODUCTION}

$\mathrm{H}$ igh-performance applications such as automotive radars require high level of integration with lower costs. The multi-layer System-on-Package (SoP) approach has emerged as an effective solution to meet these stringent needs because it has the ability of vertically integrating embedded components for additional functionality and this provides compactness and cost-savings [1]. Low temperature co-fired ceramic (LTCC) is a leading thick film technology for SoP and provides a great medium for passives integration [2]. A unique advantage of LTCC is the ability to combine materials of different dielectric constants to realize mixed LTCC tape systems. This feature enables the design to combine application specific material layers and optimize performance of individual components. Filter designs employing mixed LTCC systems have been demonstrated [3]. Though LTCC is a preferred substrate for antenna designs, a mixed LTCC tape system has never been used for antenna realization. In [4], we proposed a novel mixed LTCC tape system to demonstrate gain enhancement of an antenna module. This paper furthers our work on mixed LTCC tape systems by presenting a unique

Manuscript received April , 2011.

F. A. Ghaffar is a PhD student in Electrical Engineering Program, King Abdullah University of Science and Technology (KAUST), Thuwal 239556900, Saudi Arabia (email: farhan.ghaffar@kaust.edu.sa)

M. U. Khaled is an alumnus of Electrical Engineering Program, King Abdullah University of Science and Technology (KAUST), Thuwal 239556900, Saudi Arabia (email: muhammad.khalid@kaust.edu.sa)

K. N. Salama and A. Shamim are with Electrical Engineering Program, King Abdullah University of Science and Technolgy (KAUST), Thuwal 239556900, Saudi Arabia (email: khaled.salama@kaust.edu.sa; atif.shamim@kaust.edu.sa)
SoP with fractal antenna array and an integrated Fresnel lens.

Miniaturization of the lens and its integration with antennas at a substrate level is gaining interest $[5,6]$. A grooved Fresnel lens is a suitable candidate for such miniaturized SoP. In [5], a grooved Fresnel lens has been used for gain enhancement and beam shaping. However the lens is $15 \mathrm{~mm}$ thick, which makes it incompatible with LTCC technology. In another demonstration of enhanced gain antenna system, a $77 \mathrm{GHz}$ Yagi-Uda antenna array has been integrated with a reflector antenna [6]. A high gain (15 dBi) has been achieved with an overall size of $40 \mathrm{~mm}$ x $30 \mathrm{~mm}$ x $40 \mathrm{~mm}$. The authors of [6] recommend the use of a multi-layer substrate such as LTCC, since the MMICs can be integrated directly with the system.

This paper, for the first time, presents a $24 \mathrm{GHz}$ mixed LTCC SoP comprising an array of fractal antennas integrated with a grooved Fresnel lens. The mixed tape system allows the antenna to be realized in a relatively lower dielectric constant $\left(\varepsilon_{\mathrm{r}}=6.4\right)$ LTCC tape system and the lens to be realized in a high dielectric constant $\left(\varepsilon_{\mathrm{r}}=68.7\right)$ tape for size reduction. The lens not only enhances the array gain but also adds to the robustness of the design by providing protection against harsh conditions such as encountered in automotive radars. Unlike [5], [6] the design is simple and miniaturized, yet provides almost similar performance. The miniaturized module can easily fit into car bumpers or side mirrors etc for automotive radar applications such as blind spot detection.

\section{CONCEPTAND DESIGN}

The SoP concept is shown in Fig. 1. An aperture coupled Sierpinski Carpet fractal antenna array has been implemented on eight layers of CT707 substrate. The feed network for the antenna array is placed at the bottom of the antenna module. The feed lines excite the slots in the embedded ground plane which has been implemented on the third layer. The slots in turn, excite the fractal patch antennas which are realized on the top layer ( $8^{\text {th }}$ layer of the substrate).The fractal antenna array acts as a feed for the Fresnel lens. An air gap of $2.4 \mathrm{~mm}$ is realized between the fractal array and the lens using four corner posts to provide the necessary focal distance for the lens. The lens occupies 19 layers of CT765 substrate and enhances the system gain by $6 \mathrm{~dB}$. The design is highly suitable for integration of RF circuits with vertically integrated passives to realize compact SoP modules.

\section{A. Fractal Array}

A fractal structure is composed of numerous self-similar small units of non-integer dimensions [7]. Due to unique self 


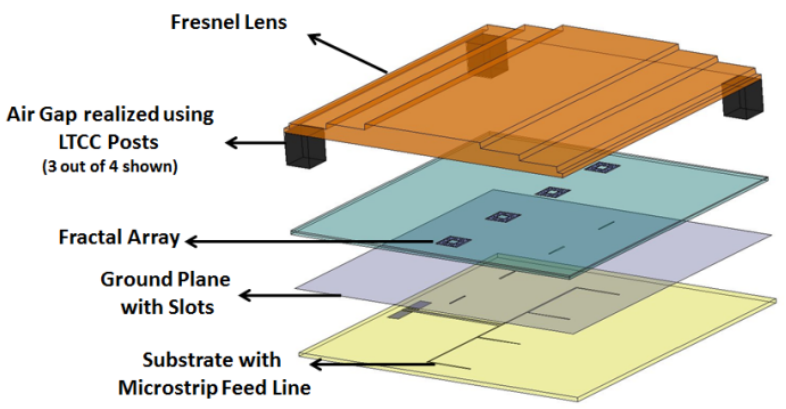

Fig. 1. SoP Concept

where $\mathrm{n}$ is the zone number, $\mathrm{f}$ is the focal distance, $\lambda_{0}$ is the free-space wavelength and $\mathrm{P}$ is the number of different phases to implement the phase correction. The focusing ability of the lens increases as $\mathrm{P}$ increases. Here $\mathrm{P}=4$ has been used as a tradeoff between the lens performance and the overall cost.

The different phases for each zone are implemented by cutting grooves into the dielectric. For a quarter wave zone plate, successive groove depths of $\mathrm{d}, 2 \mathrm{~d}$ and $3 \mathrm{~d}$ are used repetitively. The depth is given by (2) [10]:

$$
d=\frac{\lambda_{0}}{4 *\left(\sqrt{\varepsilon_{r}}-1\right)}
$$

similar shapes and structures of fractal, they provide a good platform for antennas that are compact in size and possess multiple resonances [8].The fractals provide larger bandwidths because of their space filling nature [9]. For this work a comparison has been done between a microstrip patch antenna, which is typically used as a feed for a reflector/lens antenna [5], and a fractal patch antenna. Three iterations of fractal patch antenna are investigated as shown in Fig. 2 (a). It is observed in simulations that a simple microstrip patch antenna of length $2.4 \mathrm{~mm}$ provides a bandwidth (BW) of $1.9 \%$ centered at $24 \mathrm{GHz}$, as shown in Fig. 2(b). In comparison, the increasing iterations of the fractal patch antenna (order 1-3, as shown in Fig. 2(a)) provide BWs of $2.5 \%, 4.5 \%$ and $7.5 \%$ respectively. It is worth mentioning here that the spacing between the smallest holes of the $3^{\text {rd }}$ iteration has been reduced to introduce another resonance near the centre frequency as is clear from Fig. 2(b). In addition, the fractal patch antennas from order 1 to 3 (drawn not to scale in Fig. $2(\mathrm{a})$ ), provide miniaturization of $20 \%, 32.5 \%$ and $53 \%$ as compared to conventional patch antenna respectively. The dimensions of the fractal antenna, beyond the $3^{\text {rd }}$ iteration are not suitable for LTCC fabrication. The $3^{\text {rd }}$ order fractal patch antenna $(1.8 \mathrm{~mm} \times 1.8 \mathrm{~mm})$ is selected for this work.

In order to enhance the overall gain and optimize the available space, a 4-element fractal antenna array is designed to act as a feed for the integrated lens (Fig. 1). The inter element spacing in the array is $6 \mathrm{~mm}$ which corresponds to $0.5 \lambda_{\mathrm{o}}$ at $24 \mathrm{GHz}$. The feed line of $50 \Omega$ has a width of $0.2 \mathrm{~mm}$, which is further divided into four segments each of width 0.05 $\mathrm{mm}$ to realize $200 \Omega$ impedance. The four slots placed under each element have dimensions of $0.1 \mathrm{~mm} \times 1.8 \mathrm{~mm}$. The slots are optimized to reduce the backward radiation and to provide an input impedance of $50 \Omega$. The radiation pattern of the design simulated in Ansoft HFSS is shown in Fig. 4.

B. Lens

A planar grooved Fresnel zone plate lens antenna has been designed for enhanced gain through beam shaping. The lens is a phase correcting zone plate that focuses the radiation it transmits. It consists of a set of planar zones cut into a flat piece of low loss dielectric material. The radius of each zone, $r_{n}$, is given by (1) [10]:

$$
r_{n}=\sqrt{\frac{2 n f \lambda_{0}}{P}+\left(\frac{n \lambda_{0}}{P}\right)^{2}}
$$

where $\varepsilon_{\mathrm{r}}$ is the relative dielectric constant of the material of the lens.

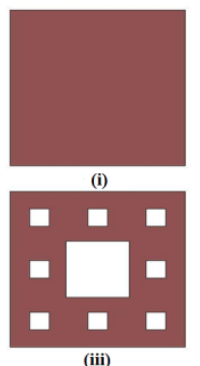

(iii)

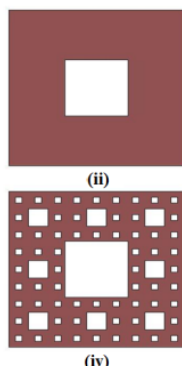

(a)

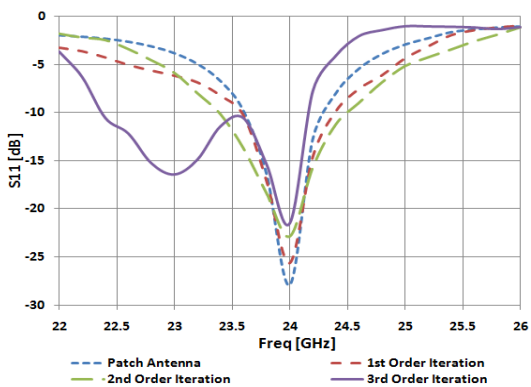

(b)
Fig. 2. Sierpinski Carpet Fractal Antenna, (a) (i) to (iv) represent iterations of order 0 to 3 respectively (drawn not to scale), (b) BW comparison with a simple patch antenna

For this work, the size of the lens has been limited to $24 \mathrm{~mm}$ x $24 \mathrm{~mm}$ to keep the SoP compact and cost effective. The commercially available CT765 LTCC tape $\left(\varepsilon_{\mathrm{r}}=68.7\right)$ has been chosen for the lens design. The high dielectric constant significantly reduces the thickness of the lens, hence minimizing the overall cost of the system which increases almost linearly with the increase in the number of layers. The focal length has been selected as $2.4 \mathrm{~mm}$ which gives a relatively low $\mathrm{F} / \mathrm{D}$ ratio of 0.1 but ensures a compact design. An optimized lens thickness of $1.6 \mathrm{~mm}$ has been achieved through simulations. Based on a single layer thickness of 85 um for the CT765 tape system, the dimensions of the grooved depths are optimized as shown in Fig. 3. Table I compares the dimensions of this design with some competing Fresnel lens reported in literature. It is clear that the previously reported designs are larger and thicker, despite the fact that they are at a higher operating frequency. The simulated results of the SoP, in Fig. 4, demonstrate that the integration of the lens results in a gain enhancement of $6 \mathrm{~dB}$ to the feed array gain, the total gain being $15 \mathrm{dBi}$ at a centre frequency of $24 \mathrm{GHz}$ in the $\mathrm{E}$ plane.

\section{MEASURED RESULTS AND ANALYSIS}

The fabrication of the SoP module is carried out in three separate parts, the fractal antenna array, the posts and the lens antenna, which are combined through post processing steps. Resbond $^{\mathrm{TM}} 989$ adhesive has been used to bond these components together. The fabricated fractal antenna array top and bottom is shown in Fig. 5(a) and 5(b) respectively. The complete fabricated SoP module is shown in Fig. 5(c).

In order to test the module through an SMA connector the ground plane must be visible. Since this is an aperture coupled 
design, the ground plane is embedded inside the SoP module. To make the ground plane accessible to the connector, it has been connected with two pads using vias as shown in Fig. 5 (b). These ground pads are placed on the bottom layer adjacent to the feed line. The provision of ground pads allows testing through an SMA connector as well as through Ground Signal Ground (GSG) RF probes.
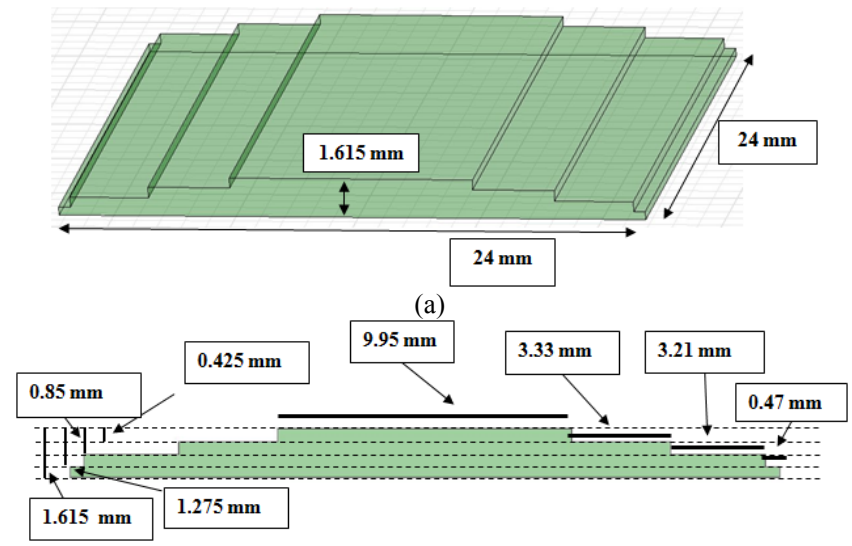

(b)

Fig. 3. (a) Lens Dimensions (b) Groove and Zone Dimensions

\begin{tabular}{|c|c|c|c|c|}
\hline References & $\begin{array}{c}\text { Centre } \\
\text { Frequency } \\
(\mathrm{GHz})\end{array}$ & $\begin{array}{c}\text { F/D } \\
\text { Ratio }\end{array}$ & $\begin{array}{c}\text { Physical } \\
\text { Dimensions } \\
(\mathrm{cm})\end{array}$ & $\begin{array}{l}\text { Thickness } \\
(\mathrm{mm})\end{array}$ \\
\hline [5] & 30 & 0.5 & 15 & 15 \\
\hline [10] & 30 & 0.5 & 15 & 15 \\
\hline [11] & 30 & 0.25 & 19.4 & 16.8 \\
\hline This Work & 24 & 0.1 & 2.4 & 1.6 \\
\hline
\end{tabular}

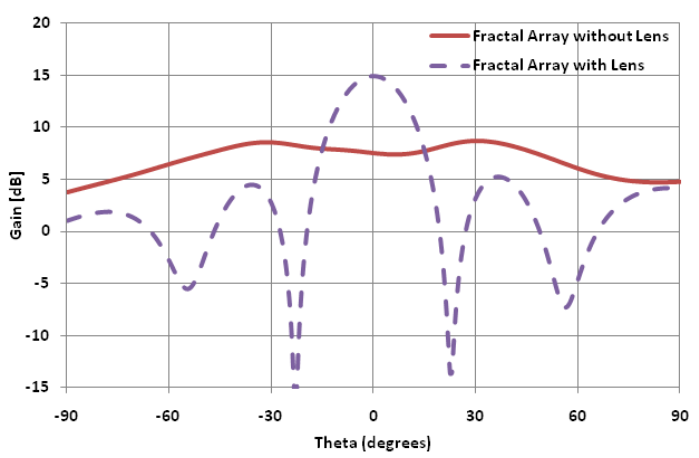

Fig. 4. Simulated Radiation Pattern of Fractal Array with and without Lens

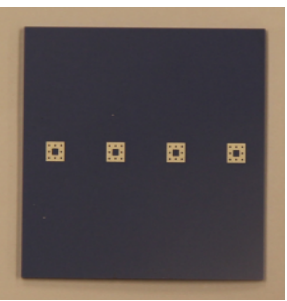

(a)

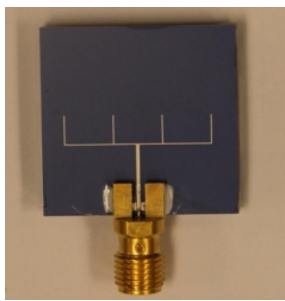

(b)

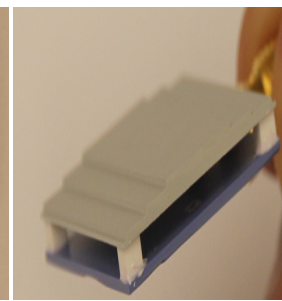

(c)
Fig. 5: Post Processing of the SoP Module

(a) Fractal Array (b) Fractal Array with SMA Connector (c) Complete SoP Module with SMA Connector

\section{A. Measurements}

The impedance measurements of the fractal antenna array without the lens are done using both an SMA connector as well as a GSG probe. Both feeding methods provide consistent results. The reflection coefficient of the array, shown in Fig. 6, depicts a shift of $1.5 \mathrm{GHz}$ in the centre frequency, making the antenna to resonate at $22.5 \mathrm{GHz}$ instead of $24 \mathrm{GHz}$. The shift in the centre frequency of the antenna array has little effect on its bandwidth performance. A measured bandwidth of 1.6 $\mathrm{GHz}$ is achieved, which is quite comparable to the simulated bandwidth of $1.8 \mathrm{GHz}$. The radiation pattern and gain measurements are done in the frequency range of $22-25 \mathrm{GHz}$, with the maximum gain achieved at $22.5 \mathrm{GHz}$. Despite the frequency shift in antenna array design, a good match is observed between the simulated and the measured radiation pattern as shown in Fig. 7. A measured gain of $9 \mathrm{dBi}$ is quite similar to the simulated gain of $8.9 \mathrm{dBi}$. The radiation pattern measurement of the antenna array is carried out at $22.5 \mathrm{GHz}$. The beam widths of $35^{\circ}$ and $140^{\circ}$ are recorded in the $\mathrm{H}$ plane and $\mathrm{E}$ plane respectively.

The measured impedance of the complete SoP is quite similar to the fractal antenna array alone. Fig. 9 shows the measured and simulated radiation patterns of the complete SoP at a centre frequency of $22.5 \mathrm{GHz}$. In measurements no enhancement to the gain of the antenna array is observed through the integration of lens. This can be attributed to a number of fabrication discrepancies and will be discussed in the next section.

\section{B. Post Measurement Analysis and Simulation}

In order to investigate the shift in the resonant frequency of the array, critical dimensions of the design have been measured. A number of discrepancies are observed in the fabricated dimensions. The fabricated antenna array has a dimension of $1.86 \mathrm{~mm} \times 1.817 \mathrm{~mm}$ as compared to the designed dimension of $1.8 \mathrm{~mm} \times 1.8 \mathrm{~mm}$. The slot has a dimension of $1.75 \mathrm{~mm} \times 0.094 \mathrm{~mm}$ instead of $1.8 \mathrm{~mm} \times 0.1$ $\mathrm{mm}$ and is also offset by $0.03 \mathrm{~mm}$ from its vertical position as shown in Fig 8(a). The dimension of the third iteration of the fractal antenna is $50 \mathrm{um}$ instead of $66.7 \mathrm{um}$ with some of the squares not etched as shown in Fig. 8 (b). In addition, the single LTCC layer thickness of the substrate is $97.5 \mathrm{um}$ which is $1.5 \mathrm{um}$ more than the designed value. This makes the total thickness of the substrate to be 780 um instead of $800 \mathrm{um}$.

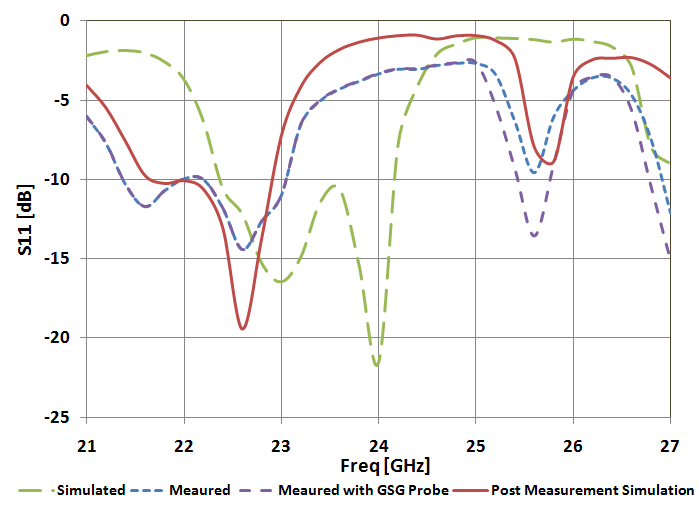

Fig. 6: Reflection Coefficient of Fractal Antenna Array

All these fabrication tolerances have been incorporated in the post measurement simulations and a shift in the resonant frequency similar to the measurements is observed. A good 
match between the measured and post measurement simulation results can be seen in Fig. 6 and Fig. 7. The post measurement simulations provide the evidence that in absence of these fabrication tolerances the original simulated results can be reproduced in the measurements.

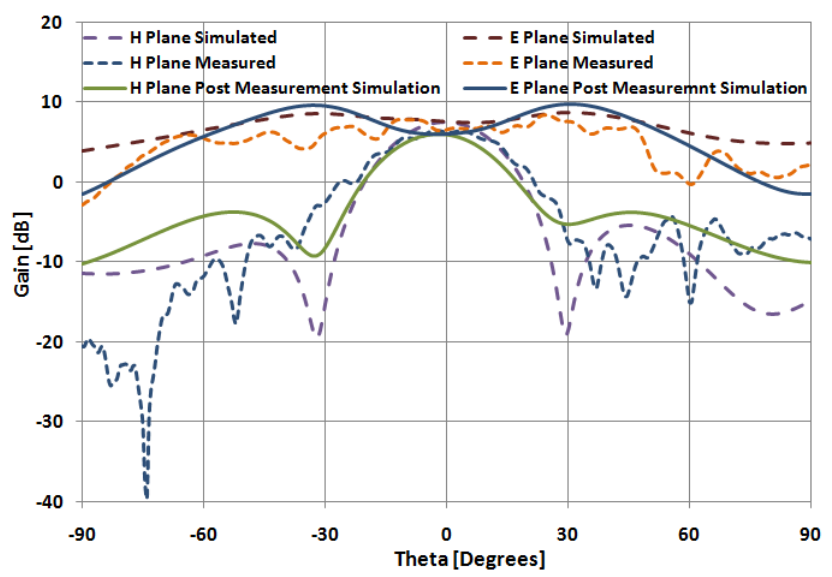

Fig. 7: Radiation Pattern of Fractal Antenna Array

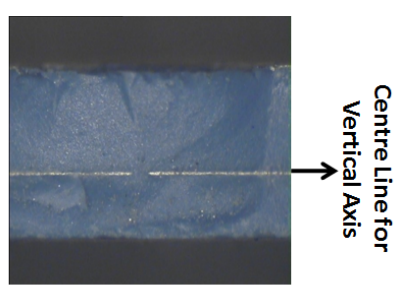

Vertical Misalignment of Slot

(a)

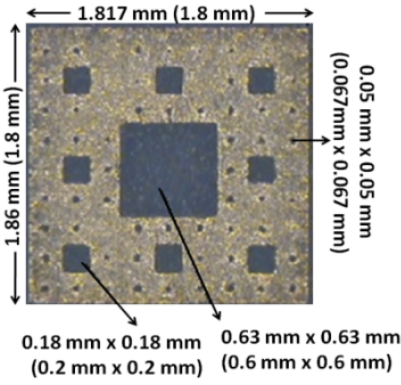

(b)
Fig. 8: Fabrication Tolerances of Fractal Antenna Array

Similar analysis on the lens reveals that the thickness of the fabricated lens is $1.56 \mathrm{~mm}$ as compared to the designed $1.615 \mathrm{~mm}$. All the groove depths have also being affected. Each zone radius of the lens has also been varied by at least $0.1 \mathrm{~mm}$. Furthermore the height of the LTCC posts is $2.33 \mathrm{~mm}$ instead of the simulated $2.4 \mathrm{~mm}$. Due to the manual post processing errors, the posts are not at the exact edges of the array substrate and are displaced by around $1.5 \mathrm{~mm}$ on average.

With the above mentioned tolerances including the dielectric constant tolerance $\left(\varepsilon_{\mathrm{r}}=68.7 \pm 0.5\right)$, the post measurement simulation results are in agreement with the measured results, as can be seen in Fig. 9. This analysis confirms that the fabrication tolerances play an important role in affecting the performance of the design.

\section{CONCLUSION}

A novel mixed LTCC tape system based SoP has been demonstrated. The SoP, for the first time, integrates a fractal antenna array with a Fresnel lens in LTCC medium to provide a gain enhancement of $6 \mathrm{~dB}$. The enhancement of the gain, though observed in simulation, could not be achieved in measurements due to some LTCC fabrication tolerances. In the absence of these tolerances the simulated gain can be achieved as evident from the post measurement simulation results. The highly integrated design is quite suitable for driver aid automotive radar applications in the $24 \mathrm{GHz}$ band. Keeping in view the fabrication tolerances of the LTCC medium, it is recommended that a patch antenna feed mechanism, such as an inset microstrip or coaxial feed, may be employed that is less sensitive as compared to the aperture coupled feed mechanism.

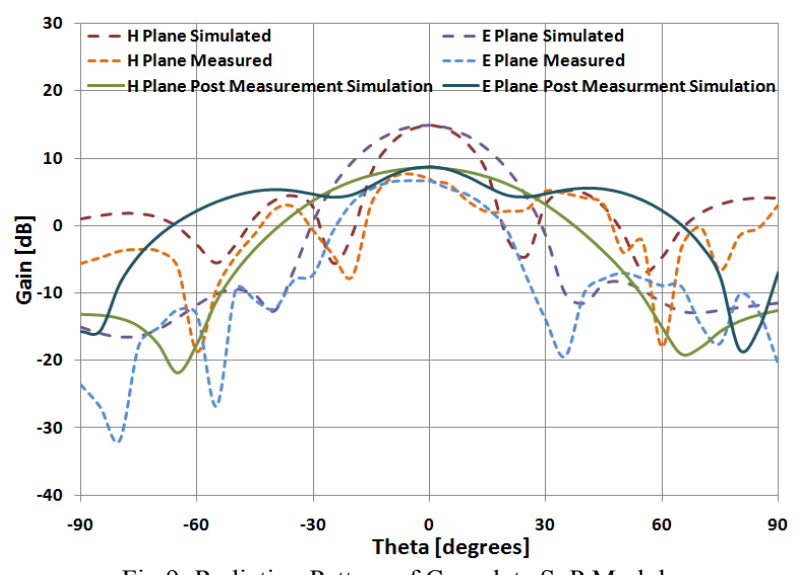

Fig.9: Radiation Pattern of Complete SoP Module

\section{ACKNOWLEDGMENT}

The authors will like to acknowledge the help of Langis Roy, Greg Brzezin from Carleton University and Aldo Petosa, Shailesh Raut from CRC Canada for the post processing and measurement of the SoP module.

\section{REFERENCES}

[1] R.R. Tummala, "The SOP for miniaturized, mixed-signal computing, communication and consumer systems for the next decade," IEEE Transactions on Advanced Packaging, vol. 27, pp. 250-267, 2004

[2] A. Shamim, et al, "Wireless Dosimeter: System-on-Chip Versus System-in-Package for Biomedical and Space Applications," Circuits and Systems II: Express Briefs, IEEE Transactions on, vol.55, no.7, pp.643-647, July 2008.

[3] A. Hennins et al, "Design Optimization and Implementation of Bandpass Filters with Normally Fed Microstrip Resonators Loaded by High-Permittivity Dielectric", IEEE Trans. Microwave Theory Tech. Vol. 54, No.3, pp. 1253-1261, Mar 2006.

[4] F.A. Ghaffar et al, "Gain-enhance LTCC System-on-Package for UMRR Applications", IEEE International Midwest Symposium on Circuits and Systems, pp. 934-937, Aug 2010.

[5] I. Kadri, A. Petosa, L. Roy, "Ka-band Fresnel Lens Antenna Fed with an Active Linear Microstrip Patch Array", IEEE Transactions on Antennas and Propagation, Vol. 53, pp. 4175-4178, Dec 2005.

[6] S. Beer, G. Adamiuk, T. Zwick, "Novel Antenna Concept for Compact Millimeter-Wave Automotive Radar Sensors", IEEE Antennas and Wireless Propagation Letters, Vol. 8, pp. 771-774, Jul 2009.

[7] D. H. Werner, S. Ganguly, "An Overview of Fractal Antenna Engineering Research", IEEE Antennas and Propagation Magazine, Vol. 45, Issue 1, pp. 38-57, 2003.

[8] A. Aggarwal, M. V. Kartikyan, "Design of Sierpinski Carpet Antenna using two different feeding mechanisms for WLAN Applications", $35^{\text {th }}$ IEEE International Conference on Infrared Millimeter and Terahertz Wave, IRMMW-Hz'10, pp.1, 2010

[9] D. P. Karmakar, D. Soren, R. Ghatak, D. R. Poddar, R. K. Mishra, "A Wideband Sierpinski Carpet Fractal Cylindrical Dielectric Resonator Antenna for X Band Application", IEEE Applied Electromagnetics Conference, $A E M C^{\prime} 09$, pp. 1-3, 2009.

[10] A. Petosa, A. Ittipiboon, "Design and Performance of a Perforated Dielectric Fresnel Lens", IEEE Proceedings-Microwaves, Antennas and Propagation, vol. 150, pp. 309-314, October 2003.

[11] A. Petosa, A. Ittipiboon, "Shadow Blockage Effects on the Aperture Efficiency of Dielectric Fresnel Lens", IEE Proceedings-Microwaves, Antennas and Propagation, Vol. 147, pp. 451-454, December 2000. 